\title{
On Chaos Behaviour of Nonlinear Lasota Equation in Lebesgue Space
}

\author{
Antoni Leon Dawidowicz ${ }^{1}$ Anna Poskrobko²
}

Received: 23 November 2019 / Revised: 29 February 2020 / Published online: 28 July 2020

(C) The Author(s) 2020

\begin{abstract}
We concern the asymptotic behaviour of the dynamical systems induced by nonlinear Lasota equation. We study chaoticity in the sense of Devaney and strong stability of the system. In many articles authors consider the properties of the linear version of the equation. By the construction of the operator in the separable space, we can formulate the relations between the solutions of the linear equation and its nonlinear version in Lebesgue space. The aim of the paper is to present the detailed construction of the operator thanks to which, the study of simple relationships allows determining the chaotic behaviour of the nonlinear equation.
\end{abstract}

Keywords Lasota equation · Dynamical system · Chaos · Stability ·

Partial differential equation

Mathematics Subject Classification (2010) 35B40 · 35B35 · 35F20 · 92B99

\section{Introduction}

Lasota equation is the part of the model of precursor cells with the assumption that cells mature with different intensity. Due to the biological application, the equation is still the matter of interest for many mathematicians. Many authors consider the linear version of the Lasota equation in various functional spaces: $C, L^{p}$, Hölder and Orlicz spaces. Despite the simple appearance of the linear equation, its asymptotic behaviour is not straightforward. Solutions show chaotic or stable behaviour depending on the value coefficient of the considered equation and parameters of the space under consideration. In some cases the question

Anna Poskrobko

a.poskrobko@pb.edu.pl

Antoni Leon Dawidowicz

Antoni.Leon.Dawidowicz@im.uj.edu.pl

1 Faculty of Mathematics and Computer Science, Jagiellonian University, ul. Łojasiewicza 6, 30-348, Kraków, Poland

2 Faculty of Computer Science, Bialystok University of Technology, ul. Wiejska 45A, 15-351, Białystok, Poland 
about the kind of the asymptotic behaviour has been left unanswered. For example, in $L^{p}$ space the decisive value of the coefficient, when we have transition from stability to chaos, is $-\frac{1}{p}$. But when we try to consider more general functional space, we do not get unambiguous results. It is known that $L^{p}$ space is the example of the Orlicz space with particular modular. Hence in paper [5], we generalize the previous results concerning $L^{p}$ space and consider Orlicz spaces with any modulars and we apply Matuszewska-Orlicz indices $p$ and $\bar{q}$ as the description of the $\varphi$ - function. And we get two critical values for the coefficient of the equation, i.e. $-\frac{1}{\underline{p}}$ and $-\frac{1}{\bar{q}}$, chaos on the interval $\left(-\frac{1}{\bar{q}}, \infty\right)$ and stability in $\left(-\infty,-\frac{1}{\underline{p}}\right]$. The question about asymptotic behaviour in the interval $\left(-\frac{1}{\underline{p}},-\frac{1}{\bar{q}}\right]$ stays still open. Chaotic behaviour of the nonlinear Lasota equation in $L^{p}$ has not yet been described. In this paper we present new results concerning this theme. We construct the operator thanks to which we can formulate the relations between the solutions of the linear Lasota equation and its nonlinear version in Lebesgue space. Thanks to this, the study of simple relationships allows determining the chaotic behaviour of the nonlinear equation. The idea of the construction is adapted from [4] where nonlinear equation in Hölder space is under consideration.

\section{Dynamical Systems}

The basic equation describing the dynamics of an age-dependent population is the McKendrick equation [13] from 1926 (called more often in the literature as the von Foerster equation). Over the years McKendrick's model was generalized in many ways, among others by Lasota and Ważewska-Czyżewska [16]. In their paper the equation is part of the mathematical description of a particular population, as the population of red blood cells is. It is the model with a feedback, because a circulatory system controls the global number of erythrocytes to some quantity optimum which can change. It happens, for example, during mountain trips or in a case of any disease of the respiratory system. Lasota, in his next paper [11], introduced a new model of precursor cells. There the main assumption is the fact that cells mature with different intensity. The form of the equation is the following

$$
\frac{\partial u}{\partial t}+c(x) \frac{\partial u}{\partial x}=f(x, u),
$$

where $c:[0,1] \rightarrow \mathbb{R}$ and $f:[0,1] \times[0, \infty) \rightarrow \mathbb{R}$ are the given functions fulfilling suitable conditions (see [12]). Here $x$ denotes the degree of cell differentiation (maturity) and the coefficient $c$ is the velocity of cell differentiation. Because of biological application, the above equation is still the matter of interest for many mathematicians $[2,8-10,14,15]$ and references therein. In this paper we consider a simpler case of the Lasota equation, that is

$$
\frac{\partial u}{\partial t}+c(x) \frac{\partial u}{\partial x}=F(u)
$$

with the initial condition

$$
u(0, x)=v(x) .
$$

We consider the semidynamical system $\left(T_{t}\right)_{t \geq 0}$ that is connected with presented problems (1)-(2) and fix our attention on its chaotic solutions in Lebesgue space $L^{p}$. We study 
chaoticity of the dynamical system in the sense of Devaney. In papers [5] and [6], we describe the asymptotic behaviour of the linear version of Lasota equation

$$
\frac{\partial u}{\partial t}+x \frac{\partial u}{\partial x}=\gamma u
$$

in $L^{p}$ space. It is known that Eq. 3 with the initial condition (2) generates the semigroup

$$
\left(\widetilde{T}_{t} v\right)=u(t, x)=e^{\gamma t} v\left(x e^{-t}\right),
$$

where $u$ is the unique solution of Eqs. 3 and 2. Asymptotic properties of the dynamical system $\left(\widetilde{T}_{t}\right)_{t \geq 0}$ depend on the value of the coefficient $\gamma$. By the construction of the operator in the separable space, we can formulate the relations between the solutions of the linear (3) and its nonlinear version (1) in Lebesgue space $L^{p}$. In Section 3, we present notation and remarks crucial in the sequel. Section 4 provides details about dynamical system connected with linear Lasota equation. In Section 5, our main results concerning the operator construction and the resulting asymptotic properties of nonlinear Lasota equation are proved.

\section{Notation, Definitions and Remarks}

Definition 1 A function $v_{0} \in V$ is a periodic point of the semigroup $\left(T_{t}\right)_{t \geq 0}$ with a period $t_{0} \geq 0$ if and only if $T_{t_{0}} v_{0}=v_{0}$. A number $t_{0}>0$ is called a principal period of a periodic point $v_{0}$ if and only if the set of all periods of $v_{0}$ is equal $\mathbb{N} t_{0}$.

\section{Definition 2 (Devaney [7])}

Dynamical system $\left(T_{t}\right)_{t \geq 0}$ defined in a metric space $(V, d)$ is chaotic as

i) $\left(T_{t}\right)_{t \geq 0}$ has a property of sensitive dependence on initial conditions in the sense of Guckenhaimer, i.e. there is a positive real number $M$ (a sensitivity constant) such that for every point $v \in V$ and every $\varepsilon>0$ there exist such $w \in B(v, \varepsilon)$ and $t>0$ that $d\left(T_{t} v, T_{t} w\right)>M$

ii) $\quad\left(T_{t}\right)_{t \geq 0}$ is transitive, that is for all nonempty open subsets $U_{1}, U_{2} \in V$ there exists $t>0$ such that $T_{t}\left(U_{1}\right) \cap U_{2} \neq \emptyset$;

iii) The set of periodic points of the system $\left(T_{t}\right)_{t \geq 0}$ is dense in $V$.

Definition 3 We define the space $L^{p, \kappa}$ to be

$$
L^{p, \kappa}([0,1])=\left\{v \in L^{p}: \int_{0}^{1}\left(|v(x)| x^{\kappa}\right)^{p} d x<\infty\right\} .
$$

If $v \in L^{p, \kappa}([0,1])$ we define the $L^{p, \kappa}$ norm of $v$ by

$$
\|v\|=\left[\int_{0}^{1}\left(|v(x)| x^{\kappa}\right)^{p} d x\right]^{\frac{1}{p}} .
$$

Remark 1 Let $m_{p, \kappa}$ be the measure on $[0,1]$ with the density $x^{p \kappa}$. Then, $L^{p, \kappa}([0,1])=$ $L^{p}\left([0,1], m_{p, \kappa}\right)$.

Remark 2 The space $L^{p, \kappa}([0,1])$ is separable. 


\section{Linear Lasota Equation}

In this section we consider the linear Lasota equation (3) with initial condition (2). In [5] and [6], it was proved that asymptotic behaviour of the dynamical system $\left(\widetilde{T}_{t}\right)_{t \geq 0}$ in $L^{p}([0,1])$, $p>0$, space depends on the coefficient $\gamma$ values. Its decisive value is $-\frac{1}{p}$.

Theorem 1 The dynamical system $\left(\widetilde{T}_{t}\right)_{t \geq 0}$ in $L^{p}([0,1]), p>0$, space is:

i) Chaotic in the sens of Devaney if $\gamma>-\frac{1}{p}$;

ii) Strongly stable if $\gamma \leqslant-\frac{1}{p}$.

Remark 3 Let us define

$$
\widehat{u}(x)=u(x) x^{\kappa} .
$$

Then, we can express the dynamical system $\left(\widetilde{T}_{t}\right)_{t \geq 0}$ in the following form

$$
\widetilde{T}_{t} u(x)=e^{\gamma t} u\left(x e^{-t}\right)=e^{\gamma t} \widehat{u}\left(x e^{-t}\right) x^{-\kappa} e^{\kappa t}=e^{(\gamma+\kappa) t} \widehat{u}\left(x e^{-t}\right) x^{-\kappa} .
$$

Therefore, we get the conclusion that in the functional space $L^{p, \kappa}([0,1])$ the dynamical system $\left(\widetilde{T}_{t}\right)_{t \geq 0}$ has the same asymptotic behaviour as the dynamical system $\left(\widehat{T}_{t}\right)_{t \geq 0}$ given by the formula

$$
\widehat{T_{t}} \widehat{u}(x)=e^{(\gamma+\kappa) t} \widehat{u}\left(x e^{-t}\right)
$$

in the space $L^{p}$.

\section{Dynamical System Generated by the Nonlinear Lasota Equation}

In the sequel we will consider the dynamical system generated by problems (1)-(2). Here, $c:[0,1] \rightarrow \mathbb{R}$ and $F:[0, a) \rightarrow \mathbb{R}$ are given continuous Lipszchitz functions satisfying conditions

$$
\begin{gathered}
c(0)=0, \quad c(x)>0 \text { for } x \in(0,1], \\
F(0)=0, \quad F(u)>0 \text { for } u \in(0, a) .
\end{gathered}
$$

If $a$ is finite, we assume also that

$$
\lim _{u \rightarrow a} F(u)=0 .
$$

From the classical theory of first-order partial differential equations, it follows that the solution of this problem has the form (see [3])

$$
u(t, x)=U(t, v(X(-t, x)))
$$

where $U(\cdot, v)$ and $X(\cdot, y)$ denote the solutions of the problems

$$
\left\{\begin{array}{l}
U^{\prime}=F(U) \\
U(0)=v
\end{array}\right.
$$

and

$$
\left\{\begin{array}{l}
X^{\prime}=c(X) \\
X(0)=y
\end{array}\right.
$$

respectively. Let a semidynamical system $T_{t}: L^{p}([0,1]) \rightarrow L^{p}([0,1])$ be given by the formula

$$
\left(T_{t} v\right)(x)=u(t, x)
$$

where $u$ is the solution of Eqs. 1 and 2 defined by Eq. 4 . 
Lemma 1 There exists increasing function $\chi:[0, \infty) \rightarrow[0, \infty)$ that its inverse function $\varphi$ fulfills the following condition

$$
\xi \varphi^{\prime}(\xi)=c(\varphi(\xi)), \quad \xi \geq 0
$$

Moreover, for every $\varepsilon>0$ there exist $\delta>0$ and $C_{1}, C_{2}>0$ such that for every $x \leq \delta$

$$
C_{1} x^{1 /\left(\lambda_{1}-\varepsilon\right)} \leq \chi(x) \leq C_{2} x^{1 /\left(\lambda_{1}+\varepsilon\right)},
$$

where $\lambda_{1}=\lim _{x \rightarrow 0} \frac{c(x)}{x}$ and $\lambda_{1} \in[0, \infty)$.

Lemma 2 For every $\gamma>0$ there exists the non-zero function $\psi:[0, a) \rightarrow(0, \infty)$ satisfying the following two conditions:

i) Firstly,

$$
\psi^{\prime}(u) F(u)=\gamma \psi(u), \quad u \geq 0 .
$$

ii) Secondly, for every $\varepsilon>0$ there exist $\delta>0$ and $D_{1}, D_{2}>0$ such that for every $u \leq \delta$

$$
D_{1} u^{\gamma /\left(\lambda_{2}-\varepsilon\right)} \leq \psi(u) \leq D_{2} u^{\gamma /\left(\lambda_{2}+\varepsilon\right)},
$$

where $\lambda_{2}=\lim _{u \rightarrow 0} \frac{F(u)}{u}$.

The detailed proofs of Lemmas 1 and 2 can be found in [3].

Theorem 2 Let $A$ be the operator defined on $L^{p}([0,1])$ space by the formula

$$
(A u)(\xi)=\psi(u(\varphi(\xi)))
$$

and let $\gamma=\frac{\lambda_{2}}{\lambda_{1}}$, where $\lambda_{1}=\lim _{x \rightarrow 0} \frac{c(x)}{x}$ and $\lambda_{2}=\lim _{x \rightarrow 0} \frac{F(x)}{x}$. Then, $A\left(L^{p}([0,1])\right)=$ $L^{q, \kappa}([0,1])$, where $q=\lambda_{1} p$ and $\kappa=\frac{1}{p}\left(1-\frac{1}{\lambda_{1}}\right)$.

Proof From Lemma 2 we have the equation

$$
\psi^{\prime}(u) F(u)=\gamma \psi(u)
$$

for some non-zero function $\psi$. Hence,

$$
\int \frac{d \psi}{\psi}=\int \frac{\gamma d u}{F(u)} .
$$

Define

$$
\Phi(x)=\left\{\begin{array}{cc}
-\int_{x}^{c_{0}} \frac{\gamma d u}{F(u)} & \text { for } x<c_{0} \\
0 & \text { for } x=c_{0} \\
\int_{c_{0}}^{x} \frac{\gamma d u}{F(u)} & \text { for } x>c_{0}
\end{array}\right.
$$

for some $c_{0} \in(0, a)$. It is obvious that

$$
\psi(x)=\psi\left(c_{0}\right) e^{\Phi(x)} .
$$

By the assumption

$$
\lim _{u \rightarrow 0} \frac{F(u)}{u}=\lambda_{2}
$$

thus for every $\eta>1$, close to 1 , there exists $\delta>0$ such that

$$
\eta^{-1}<\frac{F(u)}{\lambda_{2} u}<\eta
$$


for $u \in(0, \delta)$. For $x<\delta$

$$
\int_{x}^{c_{0}} \frac{\gamma d u}{F(u)}=\int_{x}^{\delta} \frac{\gamma d u}{F(u)}+\int_{\delta}^{c_{0}} \frac{\gamma d u}{F(u)}
$$

and

$$
\int_{x}^{c_{0}} \frac{\gamma d u}{F(u)}=\int_{x}^{\delta} \frac{\gamma d u}{F(u)}-\Phi(\delta)
$$

Moreover, it is clear that

$$
\int_{x}^{\delta} \frac{\gamma d u}{F(u)}=\frac{\gamma}{\lambda_{2}} \int_{x}^{\delta} \frac{\lambda_{2} u}{F(u)} \frac{d u}{u},
$$

so for $\lambda_{2}>0$ we have the following estimations

$$
\frac{\gamma \eta^{-1}}{\lambda_{2}}(\ln \delta-\ln x)<\int_{x}^{\delta} \frac{d u}{F(u)}<\frac{\gamma \eta}{\lambda_{2}}(\ln \delta-\ln x),
$$

and

$$
x^{\frac{\gamma \eta}{\lambda_{2}}} \delta^{-\frac{\gamma \eta}{\lambda_{2}}}<e^{-\int_{x}^{\delta} \frac{d u}{F(u)}}<x^{\frac{\gamma \eta^{-1}}{\lambda_{2}}} \delta^{-\frac{\gamma \eta^{-1}}{\lambda_{2}}}
$$

Multiplication by $\psi\left(c_{0}\right)$ gives the following inequalities

$$
\psi\left(c_{0}\right) x^{\frac{\gamma \eta}{\lambda_{2}}} \delta^{-\frac{\gamma \eta}{\lambda_{2}}}<\psi(x)<\psi\left(c_{0}\right) x^{\frac{\gamma \eta^{-1}}{\lambda_{2}}} \delta^{-\frac{\gamma \eta^{-1}}{\lambda_{2}}} .
$$

For $\lambda_{2}<0$ we have the analogical estimations but with the opposite inequalities. Let us define

$$
C(\eta)=\max \left\{\psi\left(c_{0}\right) e^{\Phi(\delta)} \delta^{\frac{\gamma \eta^{-1}}{\lambda_{2}}}, \sup _{x>\delta} \frac{\psi(x)}{x^{\frac{\gamma \eta^{-1}}{\lambda_{2}}}}\right\} .
$$

Therefore, we get

$$
\psi(x) \leq C(\eta) x^{\frac{\gamma \eta^{-1}}{\lambda_{2}}}
$$

and the conclusion

$$
\int\left|A u(\xi) \xi^{\kappa}\right|^{q} d \xi \leq C(\eta) \int|u(\varphi(\xi))|^{\frac{q \gamma \eta^{-1}}{\lambda_{2}}} \xi^{\kappa q} d \xi .
$$

By Lemma 1 we have

$$
\begin{aligned}
\int|u(\varphi(\xi))|^{\frac{q \gamma \eta^{-1}}{\lambda_{2}}} \xi^{\kappa q} d \xi & =\int|u(x)|^{p \eta^{-1}} \chi^{\prime}(x)(\chi(x))^{\kappa q} d x \leq \\
C_{2}^{\kappa q} \int|u(x)|^{p \eta^{-1}} \chi^{\prime}(x) x^{\frac{\kappa q}{\lambda_{1}}} d x & =C_{2}^{\kappa q} \int|u(x)|^{p \eta^{-1}} \chi^{\prime}(x) x^{1-\frac{1}{\lambda_{1}}} d x .
\end{aligned}
$$

The function $\chi^{\prime}$ is bounded on any interval besides zero point and

$$
\lim _{x \rightarrow 0} \frac{\chi^{\prime}(x)}{x^{\frac{1}{\lambda_{1}}-1}} \in(0, \infty) \text {. }
$$

Therefore,

$$
\int_{0}^{1}\left|A u(\xi) \xi^{\kappa}\right|^{q} d \xi<\infty
$$


Remark 4 Let a function $u$ be a solution of Eq. 1. Let us introduce the following notation $\bar{u}(\xi, t)=(A u(\cdot, t))(\xi)$. A trivial verification shows that

$$
\frac{\partial \bar{u}}{\partial t}(\xi, t)=\psi^{\prime}(u(\varphi(\xi), t)) \frac{\partial u}{\partial t}(\varphi(\xi), t)
$$

and

$$
\frac{\partial \bar{u}}{\partial \xi}(\xi, t)=\psi^{\prime}(u(\varphi(\xi), t)) \frac{\partial u}{\partial \xi}(\varphi(\xi), t) \varphi^{\prime}(\xi) .
$$

Therefore, using Lemmas 1 and 2, we get

$$
\begin{aligned}
\frac{\partial \bar{u}}{\partial t}+\xi \frac{\partial \bar{u}}{\partial \xi} & =\psi^{\prime}(u(\varphi(\xi), t))\left[\frac{\partial u}{\partial t}(\varphi(\xi), t)+c(\varphi(\xi)) \frac{\partial u}{\partial \xi}(\varphi(\xi), t)\right] \\
& =\psi^{\prime}(u(\varphi(\xi), t)) F(u(\varphi(\xi), t))=\gamma \psi(u(\varphi(\xi), t))=\gamma \bar{u} .
\end{aligned}
$$

It shows that $\bar{u}$ is the solution of Eq. 3. Therefore, the diagram

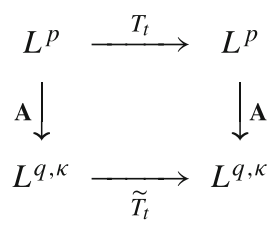

where $q=\lambda_{1} p$ and $\kappa=\frac{1}{p}\left(1-\frac{1}{\lambda_{1}}\right)$, is commutative.

Remark 5 According to Theorem 1 and Remark 3, we have chaotic behaviour of the dynamical system $\widehat{T}_{t}$ in the space $L^{q}$ if $\gamma+\kappa>-\frac{1}{q}$ and strong stability if $\gamma+\kappa \leq-\frac{1}{q}$, where $\gamma=\frac{\lambda_{2}}{\lambda_{1}}, q=\lambda_{1} p$ and $\kappa=\frac{1}{p}\left(1-\frac{1}{\lambda_{1}}\right)$.

By the above remark, we can formulate the following theorem concerning asymptotic behaviour of nonlinear Lasota equation (1).

Theorem 3 Let $\lambda_{1}=\lim _{x \rightarrow 0} \frac{c(x)}{x}$ and $\lambda_{2}=\lim _{x \rightarrow 0} \frac{F(x)}{x}$. Then, dynamical system $\left(T_{t}\right)_{t \geq 0}$ in $L^{p}([0,1])$ space is

(i) Chaotic in the sense of Devaney if $\frac{\lambda_{2}}{\lambda_{1}}>-\frac{1}{p}$;

(ii) Stable if $\frac{\lambda_{2}}{\lambda_{1}} \leq-\frac{1}{p}$.

Example 1 In paper [1] authors consider a particular form of Eq. 1, i.e.

$$
\frac{\partial u}{\partial t}+\left(x+x^{2}\right) \frac{\partial u}{\partial x}=\gamma u
$$

and prove its chaotic behaviour in $L^{p}$ space when $\gamma>-\frac{1}{p}$. However, an easy use of Theorem 3 gives the immediate conclusion, because of $\lambda_{2}=\gamma$ and $\lambda_{1}=1$.

Funding Information This work is supported by Bialystok University of Technology (Grant No. W/WI/1/2020) and founded by the resources for research by the Ministry of Science and Higher Education.

Open Access This article is licensed under a Creative Commons Attribution 4.0 International License, which permits use, sharing, adaptation, distribution and reproduction in any medium or format, as long as you give appropriate credit to the original author(s) and the source, provide a link to the Creative Commons licence, and indicate if changes were made. The images or other third party material in this article are included in the article's Creative Commons licence, unless indicated otherwise in a credit line to the material. If 
material is not included in the article's Creative Commons licence and your intended use is not permitted by statutory regulation or exceeds the permitted use, you will need to obtain permission directly from the copyright holder. To view a copy of this licence, visit http://creativecommonshorg/licenses/by/4.0/.

\section{References}

1. Abdullah HK, Haydar AK, Hussein YA. On chaotic properties of new semidynamical systems of Lasota type in two spaces. Int J Biomath. 2018;11:1850070, 15.

2. Chang $\mathrm{YH}$, Hong $\mathrm{CH}$. The chaos of the solution semigroup for the quasi-linear Lasota equation. Taiwanese J Math. 2012;16:1707-1717.

3. Dawidowicz AL, Haribash N, Poskrobko A. On the invariant measure for the quasi-linear Lasota equation. Math Methods Appl Sci. 2007;30:779-787.

4. Dawidowicz AL, Poskrobko A. Asymptotic properties of the Lasota equation in various functional spaces. AIP Conf Proc 1926. 2018;020012:79-93.

5. Dawidowicz AL, Poskrobko A. Asymptotic properties of the von Foerster-Lasota equation and indices of Orlicz spaces. Electron J Differ Eq. 2016;2016(300):1-9.

6. Dawidowicz AL, Poskrobko A. On chaotic and stable behaviour of the von Foerster-Lasota equation in some Orlicz spaces. Proc Est Acad Sci. 2008;57:61-69.

7. Devaney RL. An introduction to chaotic dynamical system. New York: Addison-Wesley; 1989.

8. Hung $\mathrm{CH}$. Chaotic and hypercyclic properties of the quasi-linear Lasota equation. Open Math. 2015;13:372-379.

9. Hung $\mathrm{CH}$, Chang YH. Frequently hypercyclic properties of the age and maturity structured model of population. Jpn J Ind Appl Math. 2019;36:461-472.

10. Kalmes T. A remark on the frequent hypercyclicity criterion for weighted composition semigroups and an application to the linear von Foerster-Lasota equation. Math Nachr. 2016;289:982-993.

11. Lasota A, Mackey MC, Ważewska-Czyżewska M. Minimizing therapeutically induced anemia. J Math Biol. 1981;13:149-158.

12. Lasota A, Szarek T. Dimension of measures invariant with respect to Ważewska partial differential equation. J Differ Eq. 2004;196:448-465.

13. McKendrick AG. Application of mathematics to medical problems. Proc Edin Math Soc. 1926;44:98130 .

14. Rudnicki R. Chaoticity and invariant measures for a cell population model. J Math Anal Appl. 2012;393:151-165.

15. Rudnicki R. An ergodic theory approach to chaos. Discrete Contin. Dyn Syst Series A. 2015;35:757-770.

16. Ważewska-Czyżewska M, Lasota A. Matematyczne problemy dynamiki układu krwinek czerwonych. Roczniki PTM Mat Stos. 1976;VI:23-40.

Publisher's Note Springer Nature remains neutral with regard to jurisdictional claims in published maps and institutional affiliations. 